\title{
TRANSPLANTE DE ÚTERO: UM CAMINHO PARA FERTILIDADE
}

\section{ARTIGO ORIGINAL}

ALENCAR, Ana Clara Silva de 1, TEIXEIRA, Gefferson Dias 2, PRAZERES, Agnes Danielle Farias ${ }^{3}$, LEITE, Mayse Zayanne Alves Gomes Vicente ${ }^{4}$, RIBEIRO, Saula Maria de Lira ${ }^{5}$, ALMEIDA, Cecília Bruna de ${ }^{6}$, MATTOS, Ludmilla Letícia Lima de ${ }^{7}$, NEVES, Izabela Carneiro ${ }^{8}$, NUNES, Thaynan Oliveira ${ }^{9}$, SANTOS, Sofia Arruda

${ }^{1}$ Discente do curso de Medicina da Universidade Ceuma.

${ }^{2}$ Médico formada pela Universidade Federal do Ceará e Residente pela UFC na Santa Casa da Misericórdia de Sobral.

${ }^{3}$ Discente do curso de Medicina da Universidade Federal do Maranhão.

${ }^{4}$ Discente do curso de Medicina do Centro Universitário UNIFACISA de Campina Grande - Paraíba.

${ }^{5}$ Discente do curso de Medicina do Centro Universitário UniFacid, Teresina-Piauí.

${ }^{6}$ Discente do curso de Medicina da Universidade de Fortaleza.

${ }^{7}$ Discente do curso de Medicina da Universidade Federal do Maranhão.

${ }^{8}$ Discente do curso de Medicina do Instituto Metropolitano de Ensino Superior Ipatinga - MG.

${ }^{9}$ Discente do curso de Medicina da Universidade José do Rosário Vellano - Alfenas $-M G$.

RC: 93251

Disponível em: https://www.nucleodoconhecimento.com.br/saude/fertilidade 
Castelo Branco ${ }^{10}$, SILVA, Eduarda Amaral da ${ }^{11}$, VASCONCELOS, Isys Holanda Albuquerque de ${ }^{12}$

ALENCAR, Ana Clara Silva de. Et al. Transplante de útero: um caminho para fertilidade. Revista Científica Multidisciplinar Núcleo do Conhecimento. Ano 06, Ed. 08, Vol. 01, pp. 152-176. Agosto de 2021. ISSN: 2448-0959, Link de acesso: https://www.nucleodoconhecimento.com.br/saude/fertilidade

\section{RESUMO}

Introdução: O transplante uterino é um procedimento cirúrgico inovador, que se encontra em fase experimental e se destina ao tratamento de pacientes com infertilidade ocasionada por fator uterino (IFU). É indicado para mulheres cujo útero não pode ser restaurado de forma cirúrgica, em pacientes histerectomizadas ou com ausência congênita do órgão, desde que atendam a determinados critérios, como a capacidade de fornecer seus próprios ovócitos e embriões, ser cariótipo XX e possuírem motivos válidos para a realização do tratamento. Objetivo: Analisar e discutir se transplante uterino é uma alternativa viável para o tratamento da infertilidade. Metodologia: Caracteriza-se como uma revisão bibliográfica do tipo integrativa, de abordagem qualitativa e natureza aplicada, objetivando destacar as perspectivas do transplante uterino para tratamento da fertilidade. A busca dos artigos foi realizada por meio do acesso à base de dados PubMed, Biblioteca Virtual e Saúde (BVS) e Scielo, realizadas no período de 2016 a 2021. Nessas buscas foram empregados os descritores, contidos no MeSH (Medical Subject Headings), relacionado à temática do artigo que inclui os termos "Transplant"; "Transplantation",

${ }^{10}$ Discente do curso de Medicina da Universidade Federal do Maranhão - Cidade Universitária - MA.

${ }^{11}$ Discente do curso de Medicina da Universidade do Sul de Santa Catarina - Palhoça $-\mathrm{SC}$.

${ }^{12}$ Discente do curso de Medicina do Centro Universitário UNINTA.

RC: 93251

Disponível em: https://www.nucleodoconhecimento.com.br/saude/fertilidade 
"Uterus"; "Fundus Uteri", "Female infertility"; "Female Reproductive Sterility" e DeCs (Descritores em Ciências da Saúde), condizentes à temática da pesquisa e pertinente aos termos: "Transplante"; "Transplantation", "Útero"; "Uterus", "Infertilidade Feminina"; "Infertility". Tais descritores, utilizados em português, inglês e espanhol e relacionados aos operadores booleanos "AND" e "OR". Resultados: Foram analisados 17 estudos, dentre eles $58,8 \%(n=10)$ demonstraram que o transplante de útero é uma boa alternativa para mulheres que sofrem com MRRH (síndrome relacionada a agenesia uterina), AUFI, síndrome de Rokitansky (que é a ausência congênita de útero) e tantos outros problemas que causam infertilidade. Os resultados também trouxeram questões éticas, religiosas e sociais $11,7 \%(n=2)$. Em contraponto, 47\% $(n=8)$ dos artigos, apresentaram dificuldades e fatores de risco para esse procedimento, principalmente por ser relativamente novo na área médica, muitas de suas consequências ainda são desconhecidas. Conclusão: Portanto, vê-se o transplante uterino como uma opção viável e com bons resultados, permitindo que pacientes inférteis possam gerar vida, somado a isso, possibilita abrir novos caminhos para discussões acerca do tema.

Palavras-chave: Transplante, Útero, Infertilidade, Tratamento.

\section{INTRODUÇÃO}

O transplante uterino é um procedimento cirúrgico inovador, que se encontra em fase experimental e se destina ao tratamento de pacientes com infertilidade ocasionada por fator uterino (IFU). Tal procedimento é indicado para mulheres cujo útero não pode ser restaurado de forma cirúrgica, em pacientes histerectomizadas ou com ausência congênita do órgão, desde que atendam a determinados critérios, como a capacidade de fornecer seus próprios ovócitos e embriões, ser cariótipo XX e possuírem motivos válidos para a realização do tratamento. Além disso, é essencial avaliar o estado psicológico da paciente, as condições de saúde e se existe a possibilidade do uso de imunossupressores (ZAAMI et al., 2018 apud CAMPOS et al., 2020). O órgão pode

RC: 93251

Disponível em: https://www.nucleodoconhecimento.com.br/saude/fertilidade 
ser proveniente de doadoras vivas ou as que tiveram, como causa de óbito, morte cerebral (KISU et al., 2018 apud CAMPOS et al., 2020).

As alternativas tradicionalmente oferecidas para o tratamento de infertilidade por fator uterino consistem em adoção e barriga de aluguel. A primeira confere a maternidade de um ponto de vista legal, enquanto a última assegura, adicionalmente, a maternidade genética (CASTELLÓN et al., 2017). Essa opção, no entanto, não é legalizada em muitos territórios, inclusive no Brasil. No país, a alternativa permitida é a gestação de substituição, ou seja, cessão temporária do útero, regulamentada pela Resolução no 2168/2017 do Conselho Federal de Medicina (CFM).

O tratamento de infertilidade por fator uterino feito com a transplantação do órgão é recente. O primeiro transplante de útero foi realizado no ano 2000, na Arábia Saudita, e envolveu uma doadora viva e uma receptora que havia sido submetida à histerectomia puerperal de emergência. Esse caso não resultou em gravidez e, após 3 meses, o útero doado foi retirado devido a uma necrose, porém não foi possível determinar a causa, uma vez que a doadora tivera uma lesão perioperatória que pode ter acarretado a incorreta perfusão do órgão. O segundo transplante foi realizado em 2011, na Turquia, dessa vez com doadora pós mortem. Dezoito meses depois, as tentativas de fertilização in vitro foram iniciadas, porém as duas gestações resultantes foram seguidas de abortamentos precoces. Novamente, o motivo do insucesso do tratamento não pôde ser determinado, visto que a doadora era nulípara, não havendo comprovação de que o útero era funcional (CASTELLÓN et al., 2017).

Com relação à fertilidade, resultados promissores vieram com um estudo clínico de coorte feito com 9 mulheres em 2013 na Suécia. A primeira gestação com nascido vivo após transplante uterino ocorreu em 2014 e foi resultado desse estudo, com pacientes que passaram por transplante de útero com doadoras vivas (BRÄNNSTRÖM et al., 2018).

A experiência sueca inspirou programas e centros de transplante uterino mundialmente, inclusive no Brasil. No país, ocorreu em 2017 a primeira gestação com RC: 93251

Disponível em: https://www.nucleodoconhecimento.com.br/saude/fertilidade 
nascido vivo após transplante de útero com doadora morta. A transferência do embrião foi feita sete meses após a transplantação, que aconteceu em 2016, e o parto cesariano ocorreu na trigésima-sexta semana de gestação, em dezembro do ano seguinte. O útero foi removido no mesmo procedimento cirúrgico (EJZENBERG et al., 2019).

Apesar dos bons resultados até então encontrados, é necessário lembrar que o procedimento ainda é experimental e possui seus riscos - além daqueles associados à gestação, complicações decorrentes do transplante e da imunossupressão são possíveis (CASTELLÓN et al., 2017). Dessa maneira, segundo Brännström et al. (2018), a temporariedade do transplante é indicada, com o planejamento de uma histerectomia, a ser realizada em "aproximadamente 5 anos, idealmente após o parto de 2 bebês saudáveis" (tradução nossa).

Ademais, questões éticas com relação à doação devem ser observadas. A preocupação com relação ao procedimento está voltada, principalmente, às doadoras vivas, uma vez que elas seriam expostas a riscos decorrentes de uma cirurgia vista como supérflua por alguns. Essa visão considera a intervenção como eletiva e nãovital, visto que não há ameaça iminente à saúde das pacientes com infertilidade por fator uterino e o tratamento não é medicamente indispensável, de forma que seria desnecessário expor as doadoras vivas aos riscos inerentes à remoção do útero (GARBUZOVA, 2021).

Assim, do ponto de vista ético, doadoras pós mortem seriam preferíveis, dada a ausência de riscos para o doador nesse caso. No entanto, desconsiderando as doadoras vivas, a disponibilidade de úteros para transplante reduz consideravelmente, tornando-se insuficiente para atender à demanda (GARBUZOVA, 2021).

Diante disso, o presente artigo surgiu com a necessidade de questionar: o transplante uterino é uma alternativa viável para o tratamento da infertilidade? Para responder esse questionamento, explorar-se-á os resultados das pesquisas atuais sobre o tema, 
de modo a abordar as perspectivas do transplante de útero para terapêutica da infertilidade como alternativa para o futuro.

\section{DESENVOLVIMENTO}

\subsection{METODOLOGIA}

O artigo em questão constitui uma revisão bibliográfica do tipo integrativa, de abordagem qualitativa e natureza aplicada, objetivando destacar as perspectivas do transplante uterino para tratamento da fertilidade.

A busca dos artigos foi realizada por meio do acesso à base de dados PubMed, Biblioteca Virtual e Saúde (BVS) e Scielo, realizadas no período de 2016 a 2021. Nessas buscas foram empregados os descritores, contidos no MeSH (Medical Subject Headings), relacionado à temática do artigo que inclui os termos "Transplant"; "Transplantation", "Uterus"; "Fundus Uter", "Female infertility"; "Female Reproductive Sterility" e DeCs (Descritores em Ciências da Saúde), condizentes à temática da pesquisa e pertinente aos termos: "Transplante"; "Transplantation", "Útero"; "Uterus", "Infertilidade Feminina"; "Infertility".

Tais descritores, utilizados em português, inglês e espanhol e relacionados aos operadores booleanos "AND" e "OR" propiciaram a origem da fórmula de busca pelo MeSH (Transplant OR Transplantation) AND (Uterus OR Fundus Uteri OR Uteri OR Uterine Cornua OR Uterine Fundus OR Uterus Cornua) AND (Female Infertility OR Female Reproductive Sterility OR Female Sterility OR Female Subfertility) e pelo DeCs (Transplante OR Transplantation OR Trasplante OR Transplantação OR Enxertia) AND (Útero OR Uterus OR Cornos Uterinos OR Cornos do Útero OR Fundo Uterino OR Fundo do Útero OR Úteros) AND (Infertilidade Feminina OR Infertility, Female OR Infertilidad Femenina OR Esterelidade Feminina).

Com o intuito de refinar a busca e aprimorar os resultados da pesquisa e critérios de elegibilidade foram empregados filtros de inclusão e exclusão. Os critérios de inclusão

RC: 93251

Disponível em: https://www.nucleodoconhecimento.com.br/saude/fertilidade 
foram os artigos que se encaixavam no tema e que poderiam ser encontrados de forma totalmente disponível e que apresentavam resumo bem estruturado. Ademais, os critérios de exclusão descartaram os artigos que não se encaixavam no tema, no qual, não estavam totalmente disponíveis e não apresentavam resumo bem estruturado.

A busca e a leitura dos artigos, em sua primeira etapa, analisaram 17 artigos, sendo, 7 da base BVSMS MEDLINE, 10 do PubMed e nenhum do Scielo, em seguida, foram colocados em uma planilha compartilhada, com sua base de dados, seu link, nome do artigo, doi do artigo e aplicados os critérios de inclusão e exclusão. Após essa etapa, restaram, 12 artigos, sendo 5 da base BVSMS MEDLINE e 7 do PubMed.

Dessa forma, vale salientar que a ordem da pesquisa não é de caráter prático, evidenciando a dispensa de submissão ao Comitê de Ética em Pesquisa (CEP). Para a apresentação dos resultados dessa pesquisa, os achados foram divididos de acordo com a temática, na qual, foram retratadas as principais informações das amostras dos artigos presentes na revisão como o título, o local, o ano de publicação, o autor, tipo de estudo, a intervenção e os principais resultados encontrados.

\subsection{RESULTADOS}

Tabela 1: Principais Resultados dos Artigos Selecionados para Pesquisa

Título

\begin{tabular}{|c|c|c|c|}
\hline & Título & $\begin{array}{l}\text { Autores, ano } \\
\text { de publicação } \\
\text { e país }\end{array}$ & Principais Resultados \\
\hline ARTIGO & $\begin{array}{l}\text { Livebirth after uterus } \\
\text { transplantation from a } \\
\text { deceased donor in a } \\
\text { recipient with uterine } \\
\text { infertility }\end{array}$ & $\begin{array}{l}\text { Ejzenberg et } \\
\text { al.,2018, Brasil }\end{array}$ & $\begin{array}{l}\text { O estudo busca } \\
\text { demonstrar a viabilidade } \\
\text { da utilização de úteros de } \\
\text { doadoras falecidas } \\
\text {,abrindo a possibilidade } \\
\text { de uma gravidez para }\end{array}$ \\
\hline
\end{tabular}

RC: 93251

Disponível em: https://www.nucleodoconhecimento.com.br/saude/fertilidade 


\begin{tabular}{|c|c|c|c|}
\hline & & & $\begin{array}{l}\text { mulheres com } \\
\text { infertilidade de fator } \\
\text { uterino, sem colocar } \\
\text { doadoras vivas em risco } \\
\text { operatório. }\end{array}$ \\
\hline $\begin{array}{l}\text { ARTIGO } \\
\text { II }\end{array}$ & $\begin{array}{l}\text { Ethical and policy issues } \\
\text { raised by uterus } \\
\text { transplants }\end{array}$ & $\begin{array}{l}\text { L. O'Donovan } \\
\text { et al., 2019, } \\
\text { Reino Unido }\end{array}$ & $\begin{array}{l}\text { O artigo em questão } \\
\text { levanta questões éticas e } \\
\text { políticas acerca do } \\
\text { transplante } \\
\text { buscando uterino, } \\
\text { impasses como o valor da } \\
\text { gestação, os riscos } \\
\text { aceitáveis } \\
\text { investimento financeiro } \\
\text { justo para esse processo } \\
\text { em contraste com os } \\
\text { outros serviços de saúde. }\end{array}$ \\
\hline $\begin{array}{l}\text { ARTIGO } \\
\text { III }\end{array}$ & $\begin{array}{l}\text { First live birth after } \\
\text { uterus transplantation in } \\
\text { the United States }\end{array}$ & $\begin{array}{l}\text { Testa } \\
\text { al,.2018, } \\
\text { Estados } \\
\text { Unidos }\end{array}$ & $\begin{array}{l}\text { O estudo demonstrou que } \\
\text { o transplante uterino pode } \\
\text { ser uma alternativa } \\
\text { terapêutica viável para } \\
\text { mulheres com } \\
\text { infertilidade uterina } \\
\text { absoluta. Ressalta-se } \\
\text { que cada caso descrito } \\
\text { acrescenta aprendizado e } \\
\text { experiência } \\
\text { clínica, aproximando, }\end{array}$ \\
\hline
\end{tabular}

RC: 93251

Disponível em: https://www.nucleodoconhecimento.com.br/saude/fertilidade 


\begin{tabular}{|c|c|c|c|}
\hline & & & $\begin{array}{l}\text { cada vez mais, o } \\
\text { procedimento de ser } \\
\text { oferecido de } \\
\text { habitual às } \\
\text { acometidas mulheres } \\
\text { disfunções que impedem } \\
\text { a gravidez. }\end{array}$ \\
\hline $\begin{array}{l}\text { ARTIGO } \\
\text { IV }\end{array}$ & $\begin{array}{lr}\text { The history } & \text { behind } \\
\text { successful } & \text { uterine } \\
\text { transplantation } & \text { in } \\
\text { humans } & \end{array}$ & $\begin{array}{l}\text { Castellón et al., } \\
\text { 2017, Brasil }\end{array}$ & $\begin{array}{l}\text { O transplante uterino tem } \\
\text { apresentado alta } \\
\text { relevância no contexto de } \\
\text { esterilidade, sobretudo } \\
\text { em pacientes com MRKH, } \\
\text { síndrome caracterizada } \\
\text { pela agenesia uterina. } \\
\text { Recomenda-se a novos } \\
\text { realização de para reforço e } \\
\text { estudos para } \\
\text { maior aplicabilidade } \\
\text { prática dos achados já } \\
\text { existentes. }\end{array}$ \\
\hline $\begin{array}{l}\text { ARTIGO } \\
\mathrm{V}\end{array}$ & $\begin{array}{l}\text { Uterine transplantation in } \\
\text { an era of successful } \\
\text { childbirths from living }\end{array}$ & $\begin{array}{l}\text { Chmel et al., } \\
2020 \text {, }\end{array}$ & $\begin{array}{l}\text { O estudo evidenciou que } \\
\text { o transplante de útero } \\
\text { tem mostrado bastante }\end{array}$ \\
\hline
\end{tabular}

RC: 93251

Disponível em: https://www.nucleodoconhecimento.com.br/saude/fertilidade 


\begin{tabular}{|c|c|c|c|}
\hline & $\begin{array}{l}\text { and deceased donor } \\
\text { uteri: Current challenges }\end{array}$ & $\begin{array}{l}\text { República } \\
\text { Tcheca }\end{array}$ & $\begin{array}{l}\text { potencial como uma } \\
\text { alternativa, } \\
\text { principalmente, para } \\
\text { mulheres com } \\
\text { infertilidade absoluta do } \\
\text { fator uterino (AUFI) que } \\
\text { desejam engravidar. }\end{array}$ \\
\hline $\begin{array}{l}\text { ARTIGO } \\
\text { VI }\end{array}$ & $\begin{array}{l}\text { Pushing the boundaries: } \\
\text { Uterine transplantation } \\
\text { and the limits of } \\
\text { reproductive autonomy }\end{array}$ & $\begin{array}{l}\text { O'Donovan , } \\
\text { 2018, Reino } \\
\text { Unido }\end{array}$ & 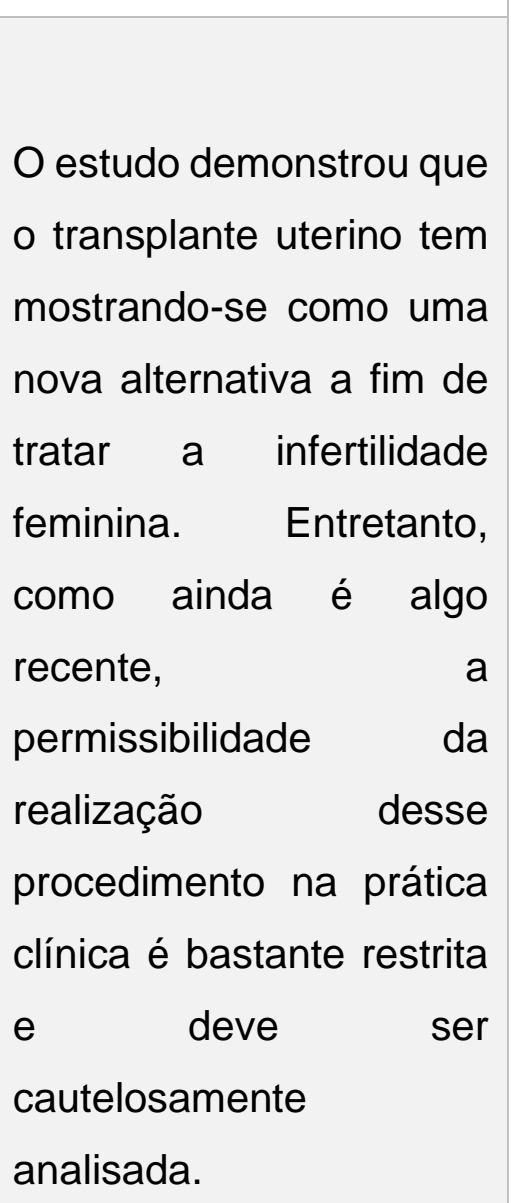 \\
\hline
\end{tabular}

RC: 93251

Disponível em: https://www.nucleodoconhecimento.com.br/saude/fertilidade 


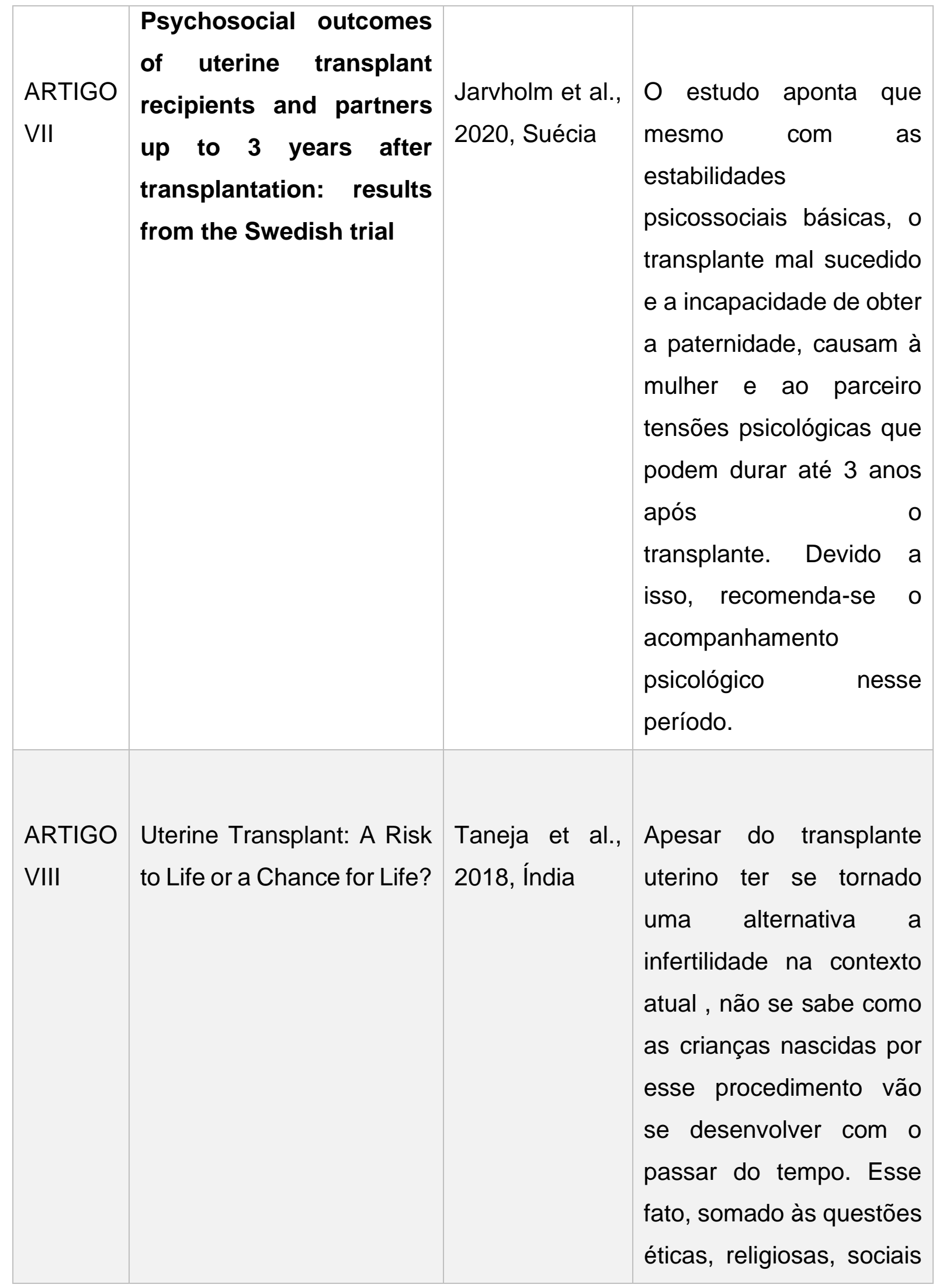

RC: 93251

Disponível em: https://www.nucleodoconhecimento.com.br/saude/fertilidade 


\begin{tabular}{|c|c|c|c|}
\hline & & & $\begin{array}{l}\text { e legais, coloca em } \\
\text { dúvida a prevalência de } \\
\text { tal método. }\end{array}$ \\
\hline $\begin{array}{l}\text { ARTIGO } \\
\text { IX }\end{array}$ & $\begin{array}{l}\text { Uterus transplantation } \\
\text { and fertility preservation }\end{array}$ & $\begin{array}{l}\text { Brannstrom e } \\
\text { Dahm-Kahler, } \\
\text { 2019, Suécia }\end{array}$ & $\begin{array}{l}\text { O estudo demonstrou que } \\
\text { existe risco aumentado } \\
\text { de neoplasias malignas } \\
\text { em pacientes que tenham } \\
\text { realizado transplante } \\
\text { uterino. } \\
\text { monitorar de forma } \\
\text { constante pacientes } \\
\text { transplantadas mediante } \\
\text { a realização de exames } \\
\text { como citologia } \\
\text { colposcopia. Prevê-se } \\
\text { ampliação desse } \\
\text { procedimento na prática } \\
\text { clinica de forma gradual } \\
\text { nos próximos anos. }\end{array}$ \\
\hline $\begin{array}{l}\text { ARTIGO } \\
x\end{array}$ & $\begin{array}{l}\text { Human uterine } \\
\text { transplantation: a review } \\
\text { of outcomes from the } \\
\text { first } 45 \text { cases }\end{array}$ & $\begin{array}{l}\text { Jones et al., } \\
2019, \\
\text { Reino Unido }\end{array}$ & $\begin{array}{l}\text { Com o nascimento bem } \\
\text { sucedido de } 18 \text { bebês } \\
\text { após o UTx, essa parece } \\
\text { ser uma alternativa para } \\
\text { mulheres com AUFI. O } \\
\text { artigo discorre também }\end{array}$ \\
\hline
\end{tabular}

RC: 93251

Disponível em: https://www.nucleodoconhecimento.com.br/saude/fertilidade 


\begin{tabular}{|c|c|c|c|}
\hline & & & $\begin{array}{l}\text { sobre a doação de útero } \\
\text { após morte cerebral. }\end{array}$ \\
\hline $\begin{array}{l}\text { ARTIGO } \\
X I\end{array}$ & $\begin{array}{l}\text { A meta-analysis on uterine } \\
\text { transplantation: Redefining } \\
\text { the limits of reproductive } \\
\text { surgery }\end{array}$ & $\begin{array}{l}\text { Silva } \\
\text { Carvalho, } \\
2016, \\
\text { Brasil }\end{array}$ & $\begin{array}{l}\text { O estudo evidenciou que } \\
\text { o transplante uterino é } \\
\text { uma inovação médica } \\
\text { que possibilita a gravidez } \\
\text { em mulheres } \\
\text { anteriormente } \\
\text { consideradas inférteis. } \\
\text { Nesse contexto, é de } \\
\text { extrema relevância a } \\
\text { discussão cautelosa das } \\
\text { vantagens } \\
\text { desvantagens } \\
\text { associadas } \\
\text { procedimento levando-se } \\
\text { em consideração todas } \\
\text { as partes envolvidas. }\end{array}$ \\
\hline $\begin{array}{l}\text { ARTIGO } \\
\text { XII }\end{array}$ & $\begin{array}{l}\text { Uterus transplant: Are we } \\
\text { close to this reality? }\end{array}$ & $\begin{array}{l}\text { Ejzenberg et } \\
\text { al., 2016, Brasil }\end{array}$ & $\begin{array}{l}\text { A infertilidade apresenta } \\
\text { relevante repercussão no } \\
\text { contexto psicossocial da } \\
\text { vida de muitas mulheres. } \\
\text { Assim, o transplante de } \\
\text { útero surge como uma } \\
\text { alternativa resolutiva }\end{array}$ \\
\hline
\end{tabular}

RC: 93251

Disponível em: https://www.nucleodoconhecimento.com.br/saude/fertilidade 


\begin{tabular}{|c|c|c|c|}
\hline & & & $\begin{array}{l}\text { nesse âmbito. No } \\
\text { entanto, como trata-se de } \\
\text { uma inovação médica } \\
\text { bastante recente, ainda } \\
\text { existem } \\
\text { obstáculos a serem } \\
\text { enfrentados e elementos } \\
\text { a serem aprimorados. }\end{array}$ \\
\hline $\begin{array}{l}\text { ARTIGO } \\
\text { XIII }\end{array}$ & $\begin{array}{l}\text { Living Donor Uterus } \\
\text { Transplant and } \\
\text { Surrogacy: Ethical } \\
\text { Analysis According to } \\
\text { the Principle of } \\
\text { Equipoise }\end{array}$ & $\begin{array}{l}\text { Testa e } \\
\text { Johannesson, } \\
\text { 2017, Estados } \\
\text { Unidos }\end{array}$ & $\begin{array}{l}\text { Para a maioria das } \\
\text { mulheres entrevistadas } \\
\text { no estudo, o desejo pela } \\
\text { gravidez supera os riscos } \\
\text { e as desvantagens } \\
\text { associadas } \\
\text { transplante } \quad \text { uterino. } \\
\text { Discorre-se também } \\
\text { sobre a maternidade } \\
\text { substitutiva gestacional } \\
\text { (GSM), em que grande } \\
\text { parte das mães de } \\
\text { aluguel aceitam os riscos } \\
\text { em detrimento do ganho } \\
\text { financeiro relacionado. }\end{array}$ \\
\hline $\begin{array}{l}\text { ARTIGO } \\
\text { XIV }\end{array}$ & $\begin{array}{l}\text { Uterine Transplantation: A } \\
\text { Survey of Perceptions and }\end{array}$ & & $\begin{array}{l}\text { Conforme o estudo, cerca } \\
\text { de } 50 \% \text { dos profissionais }\end{array}$ \\
\hline
\end{tabular}

RC: 93251

Disponível em: https://www.nucleodoconhecimento.com.br/saude/fertilidade 


\begin{tabular}{|c|c|c|c|c|c|}
\hline & $\begin{array}{l}\text { Attitudes of } \\
\text { Reproductive } \\
\text { Endocrinologists } \\
\text { Gynecologic Surg }\end{array}$ & $\begin{array}{l}\text { American } \\
\text { and } \\
\text { eons. }\end{array}$ & $\begin{array}{l}\text { Bortolet } \\
2018, \\
\text { Unidos }\end{array}$ & $\begin{array}{l}\text { to et al., } \\
\text { Estados }\end{array}$ & $\begin{array}{l}\text { médicos entrevistados } \\
\text { consideram o transplante } \\
\text { uterino como opção } \\
\text { natural e justa para } \\
\text { mulheres que possuem } \\
\text { infertilidade absoluta do } \\
\text { fator uterino. Todavia, os } \\
\text { diversos riscos atrelados } \\
\text { ao procedimento para as } \\
\text { partes envolvidas fazem } \\
\text { com que apenas uma } \\
\text { pequena parte } \\
\text { recomende esse método } \\
\text { como uma opção de } \\
\text { tratamento. }\end{array}$ \\
\hline $\begin{array}{l}\text { ARTIGO } \\
X V\end{array}$ & $\begin{array}{l}\text { A Survey of } \\
\text { Public Opinion in } \\
\text { the United } \\
\text { States } \\
\text { Regarding } \\
\text { Uterine } \\
\text { Transplantation }\end{array}$ & $\begin{array}{l}\text { Hariton } \\
\text { al.,2018, } \\
\text { Unidos }\end{array}$ & $\begin{array}{r}\text { et } \\
\text { Estados }\end{array}$ & \multicolumn{2}{|c|}{$\begin{array}{l}\text { O presente estudo conta com a } \\
\text { participação de indivíduos } \\
\text { residentes nos EUA. Nesse } \\
\text { contexto, a maioria desse público } \\
\text { é favorável a realização do } \\
\text { transplante uterino como } \\
\text { alternativa terapêutica para } \\
\text { pacientes que possuem } \\
\text { infertilidade absoluta do fator } \\
\text { uterino (AUFI), disfunção que } \\
\text { impede a gravidez da mulher. }\end{array}$} \\
\hline
\end{tabular}

RC: 93251

Disponível em: https://www.nucleodoconhecimento.com.br/saude/fertilidade 


\begin{tabular}{|c|c|c|c|}
\hline $\begin{array}{l}\text { ARTIGO } \\
\text { XVI }\end{array}$ & $\begin{array}{l}\text { Livebirth after } \\
\text { uterus } \\
\text { transplantation }\end{array}$ & $\begin{array}{l}\text { Brännström et } \\
\text { al., 2014, Suécia }\end{array}$ & $\begin{array}{l}\text { O estudo avaliou o nascimento } \\
\text { vivo de um bebê após um } \\
\text { transplante uterino de uma } \\
\text { doadora de } 61 \text { anos para uma } \\
\text { receptora de } 35 \text { anos com } \\
\text { ausência congênita do útero } \\
\text { (síndrome de Rokitansky) e } \\
\text { demonstrou a possibilidade de } \\
\text { tratamento de jovens mulheres } \\
\text { com infertilidade absoluta do fator } \\
\text { uterino por meio do enxerto de } \\
\text { útero. }\end{array}$ \\
\hline $\begin{array}{l}\text { ARTIGO } \\
\text { XVII }\end{array}$ & $\begin{array}{l}\text { Uterus } \\
\text { transplantation } \\
\text { trial: } \\
\text { Psychological } \\
\text { evaluation of } \\
\text { recipients and } \\
\text { partners during } \\
\text { the post- } \\
\text { transplantation } \\
\text { year }\end{array}$ & $\begin{array}{l}\text { Johannesson e } \\
\text { Brännström, } \\
2015 \\
\text { Reino Unido }\end{array}$ & $\begin{array}{l}\text { É necessário desenvolver uma } \\
\text { avaliação psicológica de maneira } \\
\text { sistemática e estrutural para } \\
\text { famílias que passaram pelo } \\
\text { processo de transplante uterino, } \\
\text { sobretudo, devido ao estresse } \\
\text { associado ao pós operatório do } \\
\text { procedimento. }\end{array}$ \\
\hline
\end{tabular}

Fonte: Próprio autor.

RC: 93251

Disponível em: https://www.nucleodoconhecimento.com.br/saude/fertilidade 
Dentre os estudos selecionados, aproximadamente $58,8 \% \quad(n=10)$ dos artigos demonstraram que o transplante de útero é uma boa alternativa para mulheres que sofrem com MRRH (síndrome relacionada a agenesia uterina), AUFI, síndrome de Rokitansky (que é a ausência congénita de útero) e tantos outros problemas que causam infertilidade. Brännström et al. (2014), relatou o caso de transplante realizado por doadora viva. Já Ejzenberg et al. (2018), no Brasil, foi descrito a possibilidade de bebês nascidos vivos de úteros doados por mulheres já falecidas. Assim como, Jones et al. (2019) que também teve enfoque nas doadoras falecidas após morte cerebral.

Os resultados também trouxeram questões éticas , religiosas e sociais $11,7 \%(n=2)$ como foi exposto nos estudos "Ethical and policy issues raised by uterus transplants", de L. O'Donovan et al. (2019) e também no "Uterine transplant: a risk to life or a chance for life?" de Taneja et al. (2018) .

Em contraponto, $47 \%(n=8)$ dos artigos, apresentaram dificuldades e fatores de risco para esse procedimento, principalmente por ser relativamente novo na área médica, muitas de suas consequências ainda são desconhecidas. Taneja et al., (2018) também aponta que as chances de desenvolvimento das crianças nascidas por esse procedimento ainda são desconhecidas. Já na Suécia, Brannstrom e Dahm-Kahler (2019), mesmo acreditando que o procedimento seja usado mais amplamente ao longo dos anos, destacam que há um aumento no risco de desenvolver neoplasias malignas em pacientes que se submeteram a esse transplante, ressaltando que as mesmas devem ser constantemente monitoradas com exames de citologia e colposcopia.

\subsection{DISCUSSÃO}

De acordo com os estudos de Ejzenberg et al. (2018) e Testa et al. (2018), existe e é viável utilizar úteros a partir de doadoras falecidas, e isso é importante, pois não coloca em risco a vida de doadoras vivas e ainda é uma possibilidade para mulheres que possuem infertilidade, podendo, assim, engravidar. Além disso, os casos que foram

RC: 93251

Disponível em: https://www.nucleodoconhecimento.com.br/saude/fertilidade 
descritos nos estudos relataram experiência clínica, ou seja, essa é uma forma possível de oferecer às mulheres com disfunções uma gestação saudável e com objetivo de propiciar os laços maternos. Segundo Benedet (2019), pode-se utilizar o útero de uma doadora falecida, porém é preciso avaliar se ele está capacitado para gravidez e relata que para se fazer a excisão desse órgão é preferível utilizar laparoscopia assistida a fim de tornar esse procedimento menos invasivo e considerao como uma solução aceitável, assim como um estudo sobre um grupo do Brasil, em 2017 que relatou uma gestação viável a partir de uma doadora falecida e o que parto foi bem sucedido, logo, evidencia-se a importância de investir nesse procedimento, a fim de buscar com que uma gestação saudável seja possível e viável para essas mulheres.

Conforme Castellón et al. (2017), o transplante uterino tem apresentado bons resultados e é considerado como bastante relevante no quesito esterilidade, principalmente, em mulheres que possuem MRKH, uma doença congênita em que o útero e vagina são pouco desenvolvidos ou ausentes e esta é uma das causas de infertilidade. Nesse contexto, Paiva et al. (2021) também considera o transplante de útero como uma alternativa viável e importante para mulheres acometidas com MRKH, visto que relatou que uma receptora com MRKH recebeu o útero de uma senhora de 61 anos com sucesso e o bebê nasceu com o peso de acordo com a idade gestacional e foi um caso bem-sucedido. Ainda, em concordância com esses estudos, Ejzenberg et al. (2018) relata um caso de transplante uterino em São Paulo, em 2016, em uma paciente com MRKH que recebeu de uma doadora falecida, não houve rejeição nem anormalidades com o feto durante a gestação, o bebê nasceu saudável com 36 semanas e o útero foi retirado nesse após o parto.

Em suma, os exemplos dos estudos citados demonstram que o transplante uterino é uma opção possível e uma alternativa viável para o desenvolvimento de uma gestação, além de ser um método de esperança, a fim de possibilitar que as mulheres que são inférteis possam vivenciar esse momento.

RC: 93251

Disponível em: https://www.nucleodoconhecimento.com.br/saude/fertilidade 
O fator uterino é uma condição que ocasiona o desenvolvimento da infertilidade, causada por um mioma na cavidade uterina, pólipos no endométrio ou por malformação uterina congênita, ou seja, são condições que provocam alterações na cavidade uterina tornando, assim, mais difícil a fixação. Conforme os estudos Jones et al. (2019) e Chmel et al. (2020), foi evidenciado que esse transplante tem se mostrado potencial para mulheres com infertilidade absoluta do fator uterino (IAUF), assim, se configura como uma forma alternativa para essas mulheres de terem uma gestação bem-sucedida e poderem dar à luz, além disso Campos et al. (2020) ressalta sobre a importância desse procedimento e o considera como um meio seguro para mulheres portadoras de IAUF. Desse modo, para mulheres inférteis que desejam ter um filho, todavia não por meio de barriga de aluguel, existe essa possibilidade de receber um útero de uma outra mulher visando uma gestação saudável e bemsucedida.

Assim como O'donovan et al. (2019), Garbuzova (2021) destaca a decisão entre os tipos de doadores (em vida ou falecido) como um dos principais dilemas éticos que envolvem o transplante de útero. Ao levar em conta a exposição de doadoras em vida a um procedimento invasivo e repleto de riscos, sem o propósito de salvar vidas, estudiosos fundamentados no princípio da não maleficência do Código de Ética Médica, acreditam infringir algum princípio ético com esse ato. Além do mais, a premissa da imunossupressão enquanto terapêutica pós transplante, desencoraja profissionais diante do TU ao submeter um paciente a um tratamento que não objetive salvar sua vida.

Ademais, Zaami et al. (2017) também levanta um importante dilema acerca da transplantação de um órgão reprodutor: a relação "risco X benefício", que infere justamente nas chances de sucesso ou de fracasso da operação. Embora se trate de algo muito novo em todo o mundo, no transplante de útero, assim como em qualquer outro transplante de órgãos, é importante que a receptora esteja ciente dos benefícios e dos riscos que podem se fazer presentes no seu pós-cirúrgico. Ainda que existam registros de sucesso absoluto do TU - resultando no nascimento de um bebê saudável

RC: 93251

Disponível em: https://www.nucleodoconhecimento.com.br/saude/fertilidade 
fruto de uma gestação tranquila, algumas outras possibilidades devem ser esclarecidas no momento de decidir ou não optar por essa alternativa reprodutiva, dentre elas, as chances de infecções, rejeição do órgão ou até mesmo aborto, que comprometem a eficácia da cirurgia.

Logo, tais questões acabam por sustentar a ideia de uma parcela de pesquisadores arbitrária ao TU que, baseada em princípios ultrapassados, condena o procedimento destacando e propondo alternativas mais seguras e econômicas de ter um filho. Por outro lado, nenhuma dessas outras soluções apontadas por Wilkinson et al. (2015), como a adoção e a maternidade substitutiva (barriga de aluguel), permite que a mulher sinta e tenha a experiência de gestar um filho em seu ventre. Além do mais, vale indicar que embora configure cirurgia eletiva, o transplante de útero atua como terapêutica na IAFU, contribuindo para o equilíbrio entre o bem-estar físico e mental da mulher infértil, influindo, portanto, na saúde e na qualidade de vida da população feminina afetada.

Dessa forma, Zaami et al. (2017) e Garbuzova (2021) sugerem que todos os dilemas éticos, bem como os riscos acerca do transplante sejam esclarecidos pela equipe médica, avaliados e compreendidos pela possível receptora antes de ser submetida ao procedimento cirúrgico, levando em consideração ainda as chances de o transplante não trazer os resultados esperados. Esse entendimento, somado aos investimentos e estudos acerca da temática, o TU se fortalece enquanto alternativa possível para mulheres inférteis que sonham com a chance de dar a luz ao seu bebê.

É fato que o transplante de útero é uma nova alternativa terapêutica que tem o poder de superar problemas de infertilidade de muitas mulheres. E, apesar de seus inúmeros benefícios para os pacientes e suas famílias, deve-se levar em conta possíveis impasses que poderão ser enfrentados no futuro, tanto como complicações clínicas, quanto complicações éticas que estão relacionadas ao processo do transplante. Por ser uma terapêutica recente, ainda é muito restrita a tolerância para a prática do transplante e deve-se ter muita ponderação ao considerar sua realização. Para Daolio

RC: 93251

Disponível em: https://www.nucleodoconhecimento.com.br/saude/fertilidade 
et al. (2020), faltam estudos e conhecimentos que descrevam o pós-operatório dessas pacientes, e os efeitos do transplante para as mães e para os neonatos, principalmente por conta da divergência de tempo que seriam incorridos os estudos e distinção de período operatório, pós-operatório, gestacional e neonatal.

Vale também a reflexão acerca da possibilidade de operação mal sucedida, uma vez que se criam expectativas para a paciente e sua família sobre a chance de tratamento efetivo de sua infertilidade e, assim, a realização do sonho da maternidade e/ou paternidade. Tais aspectos devem ser ponderados pela equipe médica e pela família, de preferência com acompanhamento de equipe multiprofissional para avaliação do estado psicológico dos mesmos, para evitar complicações e agravos psicológicos.

Por outro lado, mesmo com os avanços nas técnicas terapêuticas relacionadas com o processo de realização do transplante, uma vez que envolvem inúmeros riscos tanto para os doadores, quanto para os receptores.

Conforme Jones et al (2019), há inúmeras vantagens no transplante de doadoras que estão vivas, principalmente no sentido de sucesso dos procedimentos, entretanto, o número de doações é reduzido e, após as triagens e avaliações, fica ainda menor. Avalia-se então a doação de úteros após a morte cerebral da doadora, ainda de acordo com o estudo de Jones et al (2019), mesmo com as instabilidades fisiológicas, a doação após morte cerebral é uma escolha viável para muitas pacientes, porém, ainda é uma situação afetada pela redução do número de doadores - além da própria questão da instabilidade fisiológica do órgão doado. Ademais, encontram-se inúmeras barreiras no próprio processo burocrático, uma vez que o processo envolve o consentimento da família, e outros procedimentos pós e pré-operatórios, e todo esse caminho reduz gradativamente a disponibilidade para doação.

Nesse sentido, observa-se que mesmo em procedimentos que são considerados um sucesso, como o estudado por Ejzenberg et al (2018), ainda se ressalta a carência e dificuldade de encontrar doadoras, seja pela impossibilidade de doação, seja pelas 
barreiras encontradas acerca da vontade da família - principalmente nos casos de pacientes que tiveram morte cerebral.

O transplante uterino (TU) consiste em uma técnica cirúrgica capaz de permitir que, pacientes acometidas por infertilidade absoluta provocada por fator uterino (IAFU), possam gerar, gestar e dar à luz aos seus próprios filhos. Acontece que, embora seja um caminho promissor para a reprodução de mulheres inférteis, o transplante de útero é repleto de questões éticas e bioéticas que colocam em xeque o uso dessa alternativa como um progresso no campo da fertilidade.

\section{CONSIDERAÇÕES FINAIS}

Os achados dos estudos em relação à prática do transplante uterino em pacientes inférteis, tem apresentado bons resultados, sendo uma opção possível e uma alternativa viável para o desenvolvimento de uma gestação, permitindo ser um tratamento para a infertilidade de mulheres que sonham em gerar, gestar e dar a luz aos seus filhos. Essa técnica cirúrgica obedece aos critérios específicos, não sendo completamente isentas de riscos intra e pós-operatórios, e as questões éticas e bioéticas que devem ser levadas em consideração. Ressalta-se ainda, a importância de estudos futuros acerca do tema e a abertura de novos caminhos para discussões que podem auxiliar no estabelecimento dessa prática e progresso no campo da fertilidade.

\section{REFERÊNCIAS}

BENEDET, Susana. Uterus transplantation Fact sheet. Acta Obstet Gynecol Scand., v. $98, \quad$ p. 1205-1206, 25 ago. 2019. Disponível em: https://obgyn.onlinelibrary.wiley.com/doi/pdf/10.1111/aogs.13674 . Acesso em: 3 jun. 2021.

BORTOLETTO, Pietro et al. Uterine Transplantation: A Survey of Perceptions and Attitudes of American Reproductive Endocrinologists and Gynecologic RC: 93251

Disponível em: https://www.nucleodoconhecimento.com.br/saude/fertilidade 
Surgeons.. JMIG: Journal of Minimally Invasive Gynecology, Estados Unidos da América, v. 25, ed. 6, p. 974-979, 2018.

BRÄNNSTRÖM, Mats et al. Livebirth after uterus transplantation. The Lancet, Suécia, v. 385, ed. 9968, p. 607-616, 14 fev. 2015.

BRÄNNSTRÖM, Mats; DAHM-KÄHLER, Pernilla. Uterus transplantation and fertility preservation. Best Practice \& Research Clinical Obstetrics \& Gynaecology, Suécia, v. 55, p. 109-116, Fevereiro 2019.

BRÄNNSTRÖM, Mats et al. Uterus Transplantation. Transplantation, [s. I.], 2018. Disponível

em: https://journals.Iww.com/transplantjournal/Fulltext/2018/04000/Uterus_Transplantatio n__A_Rapidly_Expanding_Field.14.aspx. Acesso em: 7 jun. 2021.

CAMPOS, C. A. DE; MELOA, L. S.; RAMOSD, S.; PEREZG, S.; SILVAH, M. M.; BAPTISTAI, V. D.; FULGÊNCIOI, M.; ZERBINIJ, DE S.; SILVAP, A. M.; CARVALHO, V. E. B. de. Transplante uterino como alternativa terapêutica para infertilidade de fator uterino: uma revisão sistemática. Revista Eletrônica Acervo Saúde, v. 12, n. 10, p. e4626, 10 out. 2020.

CASTELLÓN, Luis Arturo et al. The history behind successful uterine transplantation in humans. JBRA Assisted Reproduction, Holanda, v. 21, ed. 2, p. 126-134, Abril/Maio/Junho 2017.

CHMEL , Roman et al. Uterine transplantation in an era of successful childbirths from living and deceased donor uteri: Current challenges. Biomedical Papers Med Fac Univ Palacky, República Tcheca, v. 164, ed. 1, p. 115-120, Março 2020.

DAOLIO, Jessica et al. Uterine transplantation and IVF for congenital or acquired uterine factor infertility: A systematic review of safety and efficacy outcomes in the first 52 recipients. PloS one, v. 15, n. 4, p. e0232323, 2020. Acesso em: 03 de Junho 
de

2021.

Disponível

em:

https://journals.plos.org/plosone/article?id=10.1371/journal.pone.0232323

DE CAMPOS, Camila Azalim et al. Transplante uterino como alternativa terapêutica para infertilidade de fator uterino: uma revisão sistemática. Revista Eletrônica Acervo Saúde, v. 12, n. 10, p. e4626-e4626, 2020. Disponível em: https://acervomais.com.br/index.php/saude/article/view/4626/2830. Acesso em: 3 jun. 2021.

EJZENBERG , Dani; JÚNIOR, José Maria; BARACAT, Edmund C. Uterus transplant: Are we close to this reality?. Revista da Associação Médica Brasileira, Brasil, v. 62, ed. 4, p. 295-296, Julho 2016.

EJZENBERG, Dani et al. Livebirth after uterus transplantation from a deceased donor in a recipient with uterine infertility. The Lancet, v. 392, n. 10165, p. 2697-2704, 2018. Disponível em: https://www.sciencedirect.com/science/article/abs/pii/S0140673618317665. Acesso em: 03 jun de 2021.

GARBUZOVA, E. Addressing Infertility with Uterine Transplant: An Ethical Analysis of Three Categories of Donors. Voices in Bioethics, [S. I.], v. 7, 2021. DOI: 10.52214/vib.v7i.8187.

Disponível

em:

https://journals.library.columbia.edu/index.php/bioethics/article/view/8187. Acesso em: 8 jun. 2021.

HARITON, Eduardo et al. A Survey of Public Opinion in the United States Regarding Uterine Transplantation. JMIG: Journal of Minimally Invasive Gynecology, Estados Unidos da América, v. 25, ed. 6, p. 980-985, 2018.

JÄRVHOLM, Stina et al. Psychosocial outcomes of uterine transplant recipients and partners up to 3 years after transplantation: results from the Swedish trial. Fertility and Sterility ASRM, Estados Unidos da América, v. 114, ed. 2, p. 407-415, 1 ago. 2020.

RC: 93251

Disponível em: https://www.nucleodoconhecimento.com.br/saude/fertilidade 
JÄRVHOLM, Stina et al. Uterus transplantation trial: Psychological evaluation of recipients and partners during the post-transplantation year. Fertility and Sterility ASRM, Suécia, v. 104, ed. 4, p. 1010-1015, Outubro 2015.

JONES, B. P. et al. Human uterine transplantation: a review of outcomes from the first 45 cases. BJOG: An International Journal of Obstetrics \& Gynaecology, v. 126, n. 11 ,

p.

1310-1319, 2019. Acesso em: 03 de Junho de 2021. Disp onível em: https://obgyn.onlinelibrary.wiley.com/doi/abs/10.1111/1471-0528.15863

JONES, B. P. et al. Human uterine transplantation: a review of outcomes from the first 45 cases. BJOG: An International Journal of Obstetrics \& Gynaecology, v. 126, n. 11, p. 1310-1319, 2019. Acesso em: 03 de Junho de 2021. Disponível em: https://obgyn.onlinelibrary.wiley.com/doi/abs/10.1111/1471-0528.15863

O'DONOVAN, Laura. Pushing the boundaries: Uterine transplantation and the limits of reproductive autonomy. Bioethics, Reino Unido, v. 32, ed. 8, p. 489-498, Outubro 2018.

O'DONOVAN, Laura; WILLIAMS, Nicola Jane; WILKINSON, Stephen. Ethical and policy issues raised by uterus transplants. British Medical Bulletin, Inglaterra, v. 131, ed. 1, p. 19-28, Setembro 2019.

PAIVA, Maiara Peixoto et al. O transplante de útero no tratamento da infertilidade feminina. Brazilian Journal of Development, v. 7, n. 4, p. 37790-37797, 2021. Disponível

em: https://www.brazilianjournals.com/index.php/BRJD/article/view/28108/22254. Acesso em: 3 jun. 2021.

R. C. , Luis Arturo et al. The history behind successful uterine transplantation in humans. JBRA assisted reprod., [s. I.], 2017. Disponível em: https://www.ncbi.nlm.nih.gov/pmc/articles/PMC5473706/. Acesso em: 7 jun. 2021.

RC: 93251

Disponível em: https://www.nucleodoconhecimento.com.br/saude/fertilidade 
SILVA, Ana Flávia; CARVALHO, Luiz Fernando. A meta-analysis on uterine transplantation: Redefining the limits of reproductive surgery. Revista da Associação Médica Brasileira, Brasil, v. 62, ed. 5, p. 474-477, Agosto 2016.

TANEJA, Alankrita et al. Uterine Transplant: A Risk to Life or a Chance for Life?. Science and Engineering Ethics, Índia, v. 25, ed. 2, p. 635-642, Abril 2019.

TESTA, G. et al. First live birth after uterus transplantation in the United States. American Journal of Transplantation, Estados Unidos da América, v. 18, ed. 5, p. 1270-1274, Maio 2018.

TESTA, G.; KOON, E. C.; JOHANNESSON, L. Living Donor Uterus Transplant and Surrogacy: Ethical Analysis According to the Principle of Equipoise. American Journal of Transplantation, Estados Unidos da América, v. 17, ed. 4, p. 912-916, Abril 2017.

Enviado: Junho, 2021.

Aprovado: Agosto, 2021.

RC: 93251 